\title{
Prácticas Disruptivas e Intervenciones de Diseño Desde lo Lúdico y Participativo
}

\author{
Disruptive Practices and Design Interventions from Playful and Participation
}

\author{
Diana Rodríguez Barros \\ Universidad Nacional de Mar del Plata. Argentina \\ dibarros@mdp.edu.ar
}

\begin{abstract}
We recognize creative and innovative actions in interventions from Product's Industrial Design, from the entire perspective of Design Thinking and interacting in postdigital interconnected environments. Following this framework, we present teaching practices trying to adhere to disruptive didactic practices. Specifically, we have made redesign and design interventions linking 3D models, graphics supports and prototyping. In this task, addressing working methods of solving the proyective workshop's own problems, we had used aplications of 3D NURBs modellers and meshes, hyperrealists renders and image processing. The teaching experience results, with creative responses from student's collaborative actions, have invited to established and solved unique problems in innovative ways. We consider to have developed a fruitful and meaningful experience.
\end{abstract}

Keywords: Design; Products; Practices disruptive; Teaching-learning experiences; Environment postdigital.

\section{Introducción}

En los últimos años, el Pensamiento de Diseño se ha convertido quizás en uno de los abordajes más reconocidos para identificar acciones creativas e innovadoras, en fuerte vinculación con entornos virtuales interconectados (Brown, 2009; Freire, 2012). Tales aproximaciones implican enfoques inter y multidisciplinarios, valoran la pseudo-serendipia y se expresan desde un tipo de pensamiento integrador de naturaleza abductiva que relaciona pensamiento deductivo y fiable propio de las ciencias abstractas, junto a un pensamiento inductivo y válido propio de las ciencias experimentales. En definitiva, reconoce una hibridación de pensamiento de tipo científico, tecnológico, artístico y heurístico.

Desde este enfoque, como docentes, asumimos que las tecnologías digitales no son meros dispositivos utilizados para transmitir un determinado tipo de enseñanza a nuestros estudiantes. Implican una lógica y un lenguaje que debemos aprovechar continuamente, como indica Alejandro Piscitelli (2010), una oportunidad tanto para aprender nosotros mismos como para reformular nuestras propias prácticas.

Este accionar, de respensar y redefinir constante, lo asociamos como cambios de naturaleza disruptive. Según Cristobal Cobo y John Moravec (2011), está asociado a exploraciones y experimentaciones graduales o abruptas que va produciendo modificaciones profundas que se despegan de los paradigmas tradicionales de enseñanza. Reconocemos básicamente que implica desarrollos de acciones colaborativas, diversas y complementarias, asistencia y estimulación de pensamientos convergentes y divergentes, construcción de conocimiento integrador e interpretativo, inclusión en una realidad múltiple y cambiante, actitud experimental y singular, así como verificación y validación de procesos de diseño por medio de prototipado rápido. Reconocemos también que las prácticas y las experiencias devenidas resultan mayoritariamente inmersas en un medio que ya ha dejado de ser exclusivamente digital y ha mutado hacia lo postdigital. Como bien lo precisa Hugo Pardo Kuklinky (2010), estos entornos virtuales interconectados se han ido transformando en aquellos ámbitos donde se producen transformaciones profundas y constantes especialmente a nivel de generación, producción y consumo de la información, en tanto que atraviesan y modifican nuestros campos y prácticas disciplinares desde enfoques que exceden lo meramente disciplinar.

En esta dirección enmarcamos nuestras experiencias docentes de enseñanza aprendizaje.

\section{Presentación del Caso}

Presentamos una serie de prácticas docentes experimentales, que intentamos adhieran a prácticas disruptivas (Rodríguez Barros, 2012a; Rodríguez Barros, 2012b), realizadas durante el segundo semestre del ciclo lectivo 2012 en el Taller de Informática Industrial, nivel 2 de la orientación Producto, correspondiente al 3o año de la carrera de Diseño Industrial de la Facultad de Arquitectura, Urbanismo y Diseño de la Universidad Nacional de Mar del Plata, Argentina.

Tales experiencias de enseñanza-aprendizaje desarrolladas en un ambiente de trabajo que ha valorizado lo lúdico y lo participativo, se han centrado en el diseño y gestión de bases de datos gráficos y 
de conocimiento sobre productos vinculados a juegos, en particular del tipo tablero, desde intervenciones de rediseño y prototipado en entornos postdigitales interconectados,

El curso se ha desarrollado en el ámbito del taller, asumido como modalidad didáctica que reconoce al aprendizaje proyectual desde la acción y da lugar a la resolución de problemas complejos e impredecibles desde la perspectiva del practicum reflexivo. Intervino una comisión de trabajo integrada por cuarenta y cinco estudiantes, trabajando colaborativamente en grupos de tres integrantes con responsabilidades individuales en temas referidos a modelado, gráfica y packaging del juego. Contó con la asistencia directa de un docente graduado y cuatro auxiliaries adscriptos. Ha tenido una carga horaria de cuatro horas semanales y se extendió durante doce semanas.

Intentamos, siguiendo a Donald Schön (1998), plantear situaciones pensadas y dispuestas para la tarea de enseñar a partir de prácticas del primer tipo reconociendo y aplicando técnicas y operaciones estándares, prácticas del segundo tipo reconociendo y aplicando reglas generales propias de la profesión, y prácticas del tercer tipo desarrollando y comprobando nuevas formas de conocimiento.

\section{Objetivos, Metodología y Técnicas}

Los objetivos del curso precisaron reconocer, formular y optimizar metodologías avanzadas de trabajo con modelizadores 3D NURBs y mallados en intervenciones de réplica, rediseño y diseño junto a renderizadores realísticos complementarios; explorar vínculos entre bases de datos digitales y métodos de producción; fabricar prototipos, desarrollar documentación 2D y fichas técnicas; integrar, comunicar y publicar piezas comunicacionales y envases en diferentes soportes digitales e impresos.

La metodología empleada ha recurrido a estrategias de aprendizaje propias de técnicas de resolución de problemas y proyectos (Stemberg y Spear-Swerling, 1996) Hemos enfocado el desarrollo de actividades que permitan formular situaciones problemáticas cuya resolución ha demandado analizar, descubrir, elaborar hipótesis, reflexionar, cotejar, evaluar, así como argumentar y comunicar ideas y productos, al igual que producir y verificar a los mismos.

De tal forma, sostenidas por tácticas explícitas en línea con el Pensamiento de Diseño, los estudiantes transitaron diferentes etapas de complejidad creciente, no estrictamente secuenciales ante las necesarias retroalimentaciones y simultaneidades de diseño, para desarrollar la selección y búsqueda de información sobre un juego del tipo tablero, rediseño y modelización 3D realista del producto, diseño y fabricación de los envases (packaging primarios) y soportes gráficos, así como fabricación de prototipos, testeo y prueba por parte de los estudiantes desde el rol de usuarios.

Durante la primera etapa, realizaron la búsqueda y sistematización de información sobre el objeto a intervenir; efectuaron la selección, relevamiento, registro y análisis del juego de tablero, analizando características formales, funcionales, de uso, de comunicación, de envasado y embalaje, de autoría, de usuarios, etc.; precisaron el encuadre de la intervención de rediseño; organizaron los registros del análisis de la información.

En la segunda etapa, definieron de forma general el concepto del rediseño del producto; generaron alternativas creativas para la definición del producto sobre forma, funcionamiento, tecnologías productivas y materiales, usuarios y criterios amplios de sustentabilidad; estimaron partes intangible del juego como normas y reglas, y partes tangibles del juego como rasgos de los componentes referidos a cantidad y tipo de fichas, formato del tablero de juego, escenarios, etc.; precisaron morfología y funcionamiento de los componentes; propusieron y registraron en formato $2 \mathrm{D}$ y $3 \mathrm{D}$ los lineamientos generales del producto rediseñado.

En la tercera etapa, empleando aplicaciones NURBs y superficies malladas, efectuaron el diseño y rediseño del conjunto y del detalle con modelos 3D hiperrealistas; definieron aspectos formales, utilitarios y perceptivos del juego; resolvieron conjuntos y subconjuntos, geometrías, vínculos y ensamblados entre las partes del juego; validaron la propuesta en condiciones de uso; verificaron ajustes a soluciones sustentables y factibles de fabricar; comprobaron compatibilidades dimensionales, ensamblado y montaje; y experimentaron posibilidades de modelado 2D de piezas planas y reglamentos, y modelado 3D de escenarios, piezas o fichas, tablero y envases (packaging primario), con simulación de materiales, texturas, iluminación y escenas acordes para dar apariencia real a los modelos 3D.

En la cuarta etapa, empleando aplicaciones de tratamiento de la imagen vectorial y pixelar, realizaron el diseño de los contenedores y embalajes (packaging primario y secundario) y piezas comunicacionales; definieron formal y gráficamente soportes gráficos a la manera de catálogos y afiches, definiendo la imagen identificadora del juego; resolvieron formal, gráfica y estructuralmente los envases y embalajes considerando aspectos comerciales, público al que va dirigido, canales de distribución y comercialización, junto a tipo de almacenamiento y transporte utilizado

En la quinta etapa, afrontaron la fabricación de prototipos rápidos de tablero, piezas principales y accesorias, contendedores (packaging primario), según tecnologías compatibles del tipo de corte y grabado láser, impresión offset, troquelado, y o tecnologías sustractivas de fresado CNC 2D y 3D mediante desbaste empleando cartón, espumas, acrílicos o placas de MDF según los casos; ajustaron la propuesta del diseño con documentación de planos 2D de fabricación según normativas estandarizadas para la producción del prototipo rápido; verificaron la valoración de uso, montaje y ensamble de componentes. (Ver Figura 1. Portadas presentación entorno Prezi y prototipos tableros juegos) 

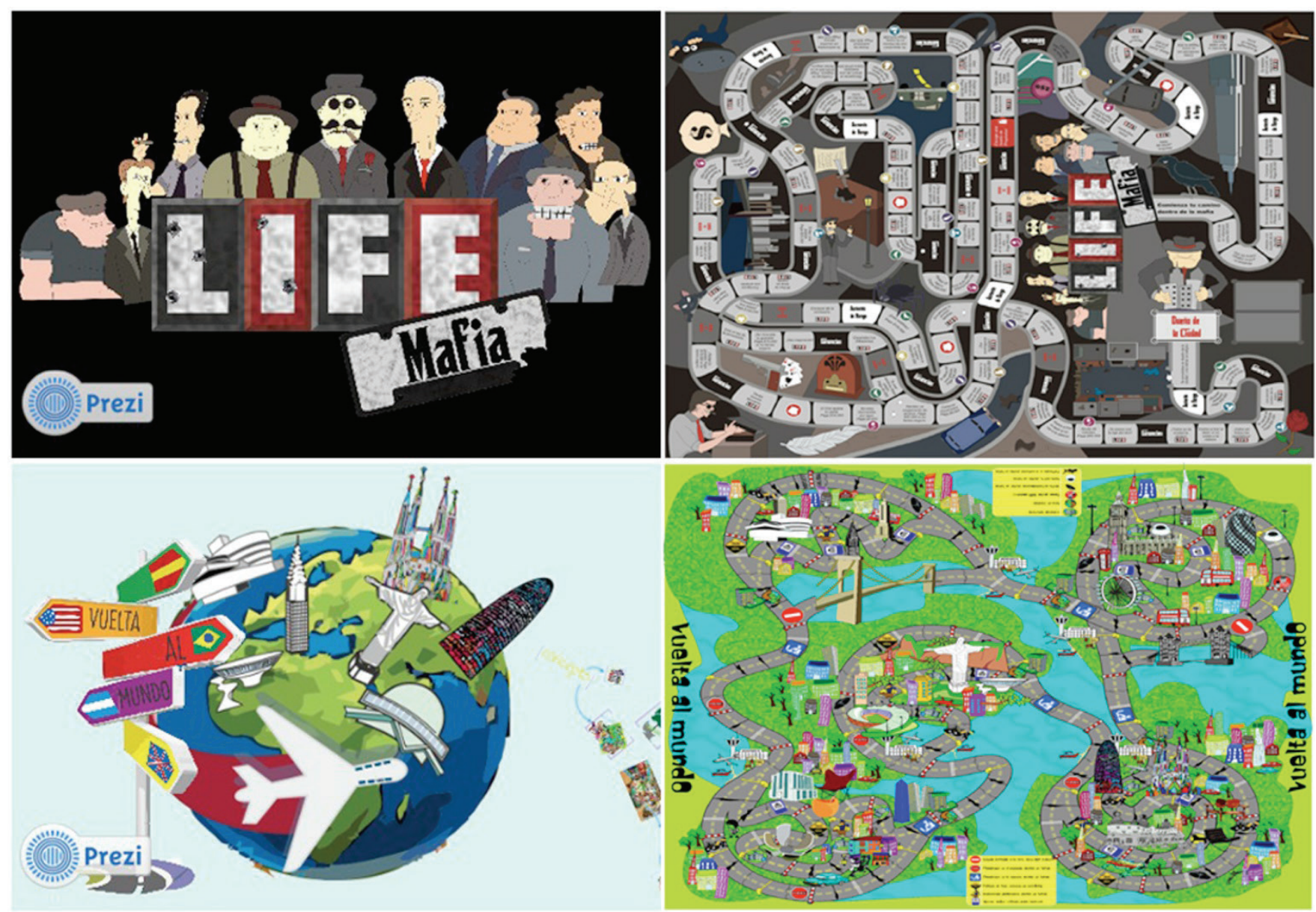

Figura 1: Portada presentación y prototipo tablero juego (2012), Life Mafia, estudiantes Rodrigo Cohen y Lucas Ruffinengo; juego Vuelta al mundo, estudiantes Amalia Ambrés y Constanza Vidart Rial. Equipo docentes Pablo Pellizonni, Guillermo Sánchez, Rodrigo Alvarez, Rodrigo Fraire, Iván Nieto

En la sexta y última etapa, los estudiantes efectuaron el testeo del juego asumiendo el rol de usuarios seleccionados y efectuaron críticas entre pares intercambiando y utilizando entre sí diversos juegos. De esta forma, retroalimentaron el proceso confrontando con premisas iniciales, evaluación de resultados, aprobación del proceso de diseño y formulación de mejoras y optimizaciones posibles.

\section{Resultados}

Hemos observado que los resultados de la experiencia en ambientes postdigitales, aunque acotada curricularmente como curso sobre aplicaciones de computación gráfica, han estimulado la posibilidad de experimentación en proyectos de diseño de productos con propuestas innovadoras generadas colaborativamente. Se actúo en un clima libre y empático de creación formal. Se valorizó practicidad, grados de utilidad y factibilidad productiva.

Hemos visto facilitado el aprendizaje, el uso de tecnologías y la transferencia a aplicaciones concretas en franca actitud exploratoria y de aprendizaje permanente. Asimismo hemos registrado que los conceptos y las metodologías generados para emprender usos, aplicaciones e interacciones de programas sobre modelizadores 3D, renderizadores y tratamiento de la imagen, han sido interpretados y entendidos a través de secuencias y consecuencias observables, en contacto directo con los objetos y con sus resultados.

Hemos verificados participación responsable junto a habilidades cognitivas de mayor grado. Como indican Christensen, Dyer y Gregersen (2011), en particular aquellas referidas a reafirmación de comportamientos deseables de observación, para identificar otras formas de acción y otras perspectivas; cuestionamiento, para desafiar los modos establecidos e interrogar otros modos posibles de acción; asociación, para generar conexión entre problemas o ideas aparentemente aisladas provenientes de campos heterogeneous; experimentación, para probar, desarmar, inspeccionar, prototipar, reflexionar y realimentar procesos. Finalmente, aintegración a redes y grupos colaborativos, para conectarse, complacerse, escuchar, establecer vínculos para descubrir otras ideas y estímulos diferentes. 


\section{Conclusiones e Implicancias}

Dada la peculiar temporalidad y obsolescencia del tipo de aplicaciones y usos sobre computación gráfica y modos de enseñanza y a partir de la observación y el reconocimiento de las necesidades de los estudiantes, hemos intentado afrontar el desafío de enseñar a usar tecnologías y transferir tales usos a aplicaciones concretas. Consideramos que tales prácticas deben estar encuadradas en una constante recontextualización y tender hacia la autogestión del conocimiento.

Asimismo asumimos que la experiencia ha sido de carácter interpretativo e integrador, opuesta al aislacionismo de los objetos tradicionales de conocimiento, restituidos en su contexto, vinculados a los observadores en calidad de usuarios comprometidos y a los lenguajes postdigitales que los sostiene y formalizan.

En particular, estimamos que la experiencia ha respondido a una construcción colaborativa tanto grupal como personal; ha estimulamos la reflexión, la búsqueda y la indagación pues los estudiantes debieron definir soluciones y estrategias que no condujeron linealmente a respuestas únicas e inmediatas, así como debieron evaluar diversas alternativas posibles formales, explorando ventajas y desventajas de los lenguajes gráficos disponibles.

A manera de conclusiones breves y provisorias, consideramos que la experiencia de aprendizaje vinculada la disenno de objetos ha generado sentido desde una serie de dimensiones de valorización fuertes. En primer lugar, la valoración de la creatividad enfocada en la capacidad transformadora en tanto idear, generar, gestionar y producir sistemas ingeniosos. En segundo lugar, la valoración de la indagación donde ha sido posible afrontar riesgos y fallas hasta obtener resultados satisfactorios y con factibilidad de transferencia hacia situaciones asimilables. En tercer lugar, la valoración de los grados de libertad, compromiso y diversión, que han estimulado desarticular y replantear reglas rígidas para fusionarlas en nuevas normas originales y practicables. Del mismo modo la valoración de entornos de trabajo participativos y cooperativos aprovechando oportunidades para interactuar con otros, ampliar los procesos implícitos, y enriquecer los resultados y tendencias.

Por tales circunstancias, continuamos trabajando en la misma dirección ampliando la experiencia en instancias de diseño, gestión y comunicación en entornos interconectados, junto a nuevas instancias exploratorias de fabricación de prototipos de los productos diseñados.

\section{Nota}

El grupo docente, bajo la dirección de la autora, ha estado integrado por el Dis.Ind. Pablo Pellizonni, contó con la colaboración de los adscriptos graduados Dis.Ind. Guillermo Sánchez y Dis.Ind. Rodrigo Alvarez junto a los adscriptos alumnos Rodrigo Fraire e Iván Nieto.

\section{Agradecimientos}

El presente escrito se encuadra en actividades de transferencia del proyecto de investigación bianual 15/B244 SCyT UNMdP 2012-2013 dirigido por la autora y radicado en los Centros CEAC y CIPADI FAUD UNMdP,

\section{Referencias}

Brown, T. (2009) Change by design: how Desgn Thinking transforms organizations and inspires innovation. Harper Collins. New York.

Christensen, C.; Dyer, J.; Gregersen, H. (2011). The innovator's DNA. Harvard Business Review Press. Boston. Ma.

Cobo, C. y Moravec, J. (2011). Aprendizaje invisible. Hacia una nueva ecología de la educación. Edicions Universitat de Barcelona. Barcelona.

Freire, J. (2012) "Ecosistemas de aprendizaje, emprendizaje e innovación" En nómada. Disponible URL http://tinyurl.com/p9rarew (consultado agosto 2013)

Pardo Kuklinski, H. (2010). Geekonomía. Un radar para producir en el postdigitalismo. Edicions Universitat de Barcelona. Barcelona.

Piscitelli, A. (2010). El Proyecto Facebook y la posuniversidad. Sistemas operativos sociales y entornos abiertos de aprendizaje. Fundación Telefónica y Ed. Ariel. Madrid y Barcelona.

Rodríguez Barros, D. (2012a). “Diseño, Enseñanza y Prácticas Disruptivas. Marcos conceptuales de referencia". En Celani, G. et.al. edits. Libro Ponencias XVI Congreso Sociedad Iberoamericana de Gráfica Digital. Universidade Federal do Ceará. Fortaleza. Pp. 251-254.

- (2012 b) "Modelado tridimensional y prototipado. Caso de aprendizaje y práctica proyectual en entorno digital". En Sciorra, J. y Valestini, S. edits. Libro Resúmenes VI Jornadas Investigación Disciplinas Artísticas y Proyectuales. Facultad Bellas Artes. Universidad Nacional de La Plata. La Plata. Pp. 31-32.

Schön, D. (1998). El profesional reflexivo. Como piensan los profesionales cuando actúan. Paidos. Barcelona.

Stemberg, R. J. y Spear-Swerling, L. (1996). "La comprensión de los principios básicos y de las dificultades de enseñar a pensar". En Enseñar a pensar. Santillana. Madrid. Pp.95-118. 